\title{
Current concepts in management of chronic wounds
}

\author{
Chandrasinghe P.C, MBBS ${ }^{1}$, Ariyaratne M.H.J, MBBS, MS, FRCS(Ed.\&Eng.). ${ }^{2}$ \\ 1 Surgical Registrar, University Surgical Unit, North Colombo Teaching Hospital, Ragama. \\ 2 Professor in Surgery, University Surgical Unit, North Colombo Teaching Hospital, Ragama.
}

Key words: Wounds; Biofilm.

\section{Introduction}

A wound is defined as a disruption in the normal anatomic structure and function. The human body has an innate ability to heal wounds; the process was described by James Moore (Surgeons' Company of London: 1789) as "vis medicatrix naturae"[1]. The clinician plays a supportive role in providing a facilitatory environment for wounds to heal. The modern concepts of wound bed preparation, which originated from the principles of Ambrose Pare (1585) and Guy de Chauliac (1365) focus on removal of devitalised tissue, reducing bacterial burden, maintaining moisture and stimulating epithelialisation. Wounds are classified as acute or chronic based on whether an orderly and timely healing process has taken place to restore continuity and function. The focus of this article will be on chronic wounds.

\section{The process of wound healing}

Wound healing consists of four overlapping phases of haemostasis, inflammation, proliferation and remodelling. During the haemostasis phase, while the clot is being formed, the activation of platelets plays a major role in initiating wound healing. Transforming growth factor $\beta$ (TGF- $\beta)$ and platelet derived growth

Correspondnce: M. H. J. Ariyaratne, Professor in Surgery, University Surgical Unit, North Colombo Teaching Hospital, Ragama.

Email: surgerydptuk@yahoo.com

The Sri Lanka Journal of Surgery 2010; 28(2):2-5. factor (PDGF) secreted by platelets, attract neutrophils, macrophages and fibroblasts.

The inflammatory phase is dominated by neutrophils, and later, by macrophages. Neutrophils secrete Interleukine-1 (IL-1) and tumour necrosis factor (TNF), while the macrophages secrete PDGF, TGF-B, TNF-A, fibroblast growth factor (FGF), insulin like growth factor-1 and IL-1 which stimulate fibroblasts and epithelial cells. The remodelling phase is dominated by fibroblast activity which aims to achieve tissue continuity. Type III collagen which is seen in the initial phase is now being replaced by type I collagen which gives a maximum tensile strength of about $80 \%$ of the normal tissue. Recent interest has been drawn towards zinc dependant matrix metalloproteinases (MMP) which are regulated by TGF- $\beta$. These MMPs control the degradation of the wound matrix to allow epithelial migration, angiogenesis and overall remodelling [2] and are found in higher amounts in chronic wounds. Through this pathway TGF- $\beta$ plays a central role in scar formation. A wound is categorised as chronic when it fails to progress as expected and fails to heal after 30 to 60 days [3]. All chronic wounds begin as acute wounds but various patient related and external factors, which alter the normal cellular and molecular response, cause them to become chronic by deviating from the expected progression explained above.

\section{Colonization, infection and biofilms}

A wound is considered to be infected, as opposed to being colonized, when a live 
microbial cell count of more than $10^{5}$ organisms is demonstrated [4]. Pathway from colonization to infection was previously thought to be linear. Our understanding of biofilms has complicated this model. Biofilms are found in chronic wounds and are rarely, if ever, seen in acute wounds. Biofilm is an aggregation of microbes belonging to several species of bacteria that manufacture a protective carbohydrate matrix which allows them to adhere to each other and to a host wound surface [5]. A wound with a biofilm will not show the classical signs of inflammation, but will carry a live microbial cell count greater than $10^{5}$. Presence of a biofilm will require intervention as the host will not be able to overcome the burden without surgical debridement. Presence of a biofilm acts as a mechanical impedance to wound healing while it deregulates the inflammatory process by affecting intracellular signalling [4].

\section{Wound bed preparation}

Although wound bed preparation is an accepted approach to chronic wound management, the need for optimising systemic factors in the patient cannot be overemphasised. Adequate blood supply, correction of haemoglobin, nutrition, glycaemic control and trace elements such as vitamin $\mathrm{C}$ and zinc are vital. Wound bed preparation is aimed at de-sloughing, reducing bacterial burden, maintaining moisture and stimulating the epidermal cell migration from the edge. This concept is popular under the acronym TIME [7]- T-tissue debridement, Iinfection control, M-maintaining moisture, Eadvancing edge.

\section{Debridement}

Wound debridement is aimed at converting the wound bed to that found in an acute wound. It removes necrotic tissue that provides a culture medium for bacteria, pockets of infection, microbes along with the bio-film, tissue debris that interfere with cell migration and senescent cells [7]. The term 'debridement' was used liberally by surgeons until 1996 when it was standardised by the International Advisory board of Wound Management. It classified debridement into five numerical categories allowing proper documentation and communication [8](Table 1). The removed tissue is also described as: skin (S), subcutaneous connective tissue (C), deep soft tissue/ muscle/nerves/vessels (M) and bone (B). This allows an alphanumerical classification. Hydrodissection is a recent advancement which allows a complete or radical debridement with the use of a water jet. It cuts and aspirates necrotic tissue. Furthermore, pulsed electric and radiofrequency stimulation have also shown faster wound healing rates [9]. Ultrasound and pressureirrigation have been tried with poor results.

Table 1. Classification of wound debridement by the International Advisory Board of Wound Management

\begin{tabular}{lll} 
Category & Description \\
\hline 0 & Non Debrided & $\begin{array}{l}\text { Not undergone TIME based } \\
\text { preparation }\end{array}$ \\
1 & Incomplete & $\begin{array}{l}\text { All non viable tissue were not } \\
\text { removed } \\
\text { Removal of obvious nonviable } \\
\text { tissue } \\
\text { Removing non viable and } \\
\text { potentially viable tissue } \\
\text { Complete debridement plus a ring } \\
\text { of normal tissue }\end{array}$ \\
\hline
\end{tabular}

\section{Biofilm eradication}

There are several methods of mechanical and chemical wound debridement used to eradicate the biofilm. Conventional surgical desloughing and the newer methods described above are all effective. The sterile larvae of Lucilia sericata (green bottle fly) has proven to be effective by removing only the non viable tissue and bacteria including strains of Staphylococcus aureus while its secretions are known to stimulate granulation tissue formation by stimulating fibroblasts [10]. The antimicrobial properties of silver, by inhibition of electron transport in bacteria, are used in modern dressings. Iodine in the form of 'cadexomer iodine' has shown to be effective against biofilms [11]. Two other chemicals; triclosan and gallium, have been found to be capable of inhibiting biofilm formation in-vitro. Leptospermum honey produced from the tree 
belonging to the genus Leptospermum contains methylgyoaxal which acts against biofilms and many other microbes including methicillin resistant staphylococcus aureus (MRSA) [12]. This is a good option for resource-poor settings. Topical antibiotics are ineffective as bacteria in biofilms are more than one thousand times resistant compared to planktonic culture.

\section{Maintaining moisture}

A controlled amount of moisture is required in the wound bed for optimal healing [13]. Hydrogel and hydrocolloid dressings will keep a wound bed moist allowing the phagocytes to continue a selective debridement. Semipermeable polyurethane membranes are used to retain moisture while allowing heat and gas exchange. Calcium alginate containing dressings are used to absorb excess exudate from wounds. Collagen containing dressings are designed to retain moisture while binding to factors that inhibit wound healing (eg: proteases). Negative pressure wound therapy (NPWT) has gained popularity in the recent past due to its ability to control moisture in exudative wounds and aid wound contraction. Though this type of dressing has shown significant results in trials, the overall evidence in this regard is conflicting [14].

\section{Skin cover}

There is a wide range of synthetic, allogenic, autologous and tissue of animal origin available for skin cover. Synthetic covers are mainly made of silicone and collagen. Bovine and porcine tissues have been used as xenografts. Human cadaveric skin and bioengineered neonatal human fibroblasts and keratinocytes are also used as temporary skin cover with good results [15]. Autologous grafts from cultured keratinocytes, fibroblasts and stem cells are available [16] eliminating the hazard of disease transmission seen in allografts and xenografts.

\section{Venous Ulcers}

Venous ulcers merit special mention under chronic ulcer management. Apart from factors mentioned above, compression therapy and superficial venous surgery play a centre role in the healing of venous ulcers. Compression therapy with four layered bandage using a pressure of $40 \mathrm{~mm} \mathrm{Hg}$ at the ankle has proven to achieve faster healing [17]. The ESCHAR (Effect of surgery and Compression on Healing and Recurrence in patients with chronic venous ulcers) study provides evidence that superficial venous surgery, when combined with compression, reduces recurrence of venous ulcers compared to compression alone [18].

Management of chronic wounds is a challenging for the surgeon. Understanding the physiology of wound healing and utilising advancements in wound care to optimise the wound bed will help to overcome this challenge in a rewarding manner.

\section{References}

1. Moore JC. A dissertation on the process of nature in the filling up of cavities, healing of wounds, and restoring parts which have been destroyed in the human body. John Richardson printer to the Lyceum Medicum Londinense; 1789.

2. Chen WY, Rogers AA, Lydon MJ. Characterization of biologic properties of wound fluid collected during early stages of wound healing. Journal of Investigatory Dermatology 1992; 99(5): 559-64.

3. James GA, Swogger E, Wolcott R et al. Biofilms in chronic wounds. Wound repair and regeneration 2008; 16(1): 37-44

4. Rhoads DD, Wolcott RD, Percival SL. Biofilms in wounds: Management stratergies. Journal of Woundcare 2008; 17 (11):502-8.

5. Donald RM, Costerton JW. Biofilms: Survival mechanisms of clinically relevant microorganisms. Clinical Microbiology Review 2002; 15(2):167-93.

6. Shultz GS, Mozingo D, Romanelli M et al. Wound healing and TIME; New concepts and scientific applications. Wound repair and regeneration 2005; 13 (suppl 4):S1-11.

7. Steel DL, Donahue D,Webster MW et al. Effect of extensive wound debridement and treatment on the healing of the 
diabetic foot ulcers. Journal of American College of Surgeons 1996; 183:64.

8. Granick MS, Chehade M. The evolution of surgical wound management: Towards a common language. Surgical wound healing and management. New York: London. Informa Healthcare; 2007. P1727.

9. Feedar JA, Kloth LC et al. Chronic dermal ulcer healing with monophasic pulsed electrical stimulation. Physical Therapy 1991; 71:639-649.

10. R.A. Sherman, M.J.R. Hall, S. Thomas. Annual Review of Entomology 2000; 45:55-81.

11. Akiyama H, Oono T, Saito M. Iwatsuki K. Assessment of cadexomer Iodine against S.aureus biofilm in vivo and invitro using confocal laser scanning microscopy. Journal of Dermatology 2004; 31(71):52934.

12. Clutterbuck AL, Cochrane AC, Dolman J, Percival SL. Evaluating antibiotics to use in medicine using a poloxamer biofilm model. Annals of Clinical Microbiology and Antimicrobials 2007; 6:2.
13. Hinman CD, Maibach HI. Effect of air exposure and occlusion on experimental human skin wounds. Nature 1963; 200:377-8.

14. Sven Gregor, Marc Maegele et al. Negative Pressure Wound Therapy: A Vacuum of Evidence? Archives in Surgery. 2008;143(2):189-196.

15. Schurr MJ et al. Phase I/II clinical evaluation of Strata Graft: A consistent, pathogen free human skin substitute. Journal of Trauma 2009; 66:866 - 74 .

16. Hefton JM, Caldwell D, Biozes DG, Balin AK, Carter DM. Grafting of skin ulcers with cultured autologous epidermal cells. Journal of the American Academy of Dermatology 1986; 14:399-405.

17. Cullum M, Nelson ED, Fletcher DW et al. Compression for venous leg ulcers. Cochrane Database Systematic Review 2001; 2:CD 000265

18. Barwell JR, Taylor M, Chant T, Foy C et al. Comparison of surgery and compression with compression alone in chronic venous ulceration (Eschar study); Randomised Controlled Trial. Lancet 2004; 363:18549. 